\title{
IGA IIPG for Elasticity Problems on Overlapped Patches
}

\author{
Honghai Zhang, Rong Mo and Neng Wan \\ Key Laboratory of Contemporary Design and Integrated Manufacturing Technology, Ministry of Education, Northwestern \\ Polytechnical University, Xi’an, China, 710072
}

\begin{abstract}
IGA(IsoGeometric Analysis) is developed to integrate the CAD and FEA process. If the union operator is applied in the CAD process, the overlapped parts should be trimmed to carry out the analysis. Therefore, to keep the integration precision on the trimmed patches, some timeconsuming algorithms should be applied. In this paper, an IIPG (incomplete interior penalty method) is applied to solve the elasticity problems defined on the overlapped domains. With this method, the time-consuming integration processes on the trimmed patches are avoided.
\end{abstract}

Keywords-isogeometric analysis; discontinuous galerkin method; overlapped domain; elasticity

\section{INTRODUCTION}

The Isogeometric Analysis (IGA ,Hughes et al.[1]-[4]) is developed to eliminate the gap between the CAD and FEA. It applies the same basis to describe both the geometries and the unknown fields. With IGA, the geometries are kept unchanged exactly[4], [5] in the refinement process. Now, IGA has been successfully applied to solve many physical problems, such as the elasticity, the electromagnetism, the fluid-structure interaction, and the shape and topological optimization problems. In the CAD modeling process, designers often use the Boolean operators to create new geometries. When the union operator is applied on some overlapped parts to create new CAD models, some of the IGA methods[6]-[8] need to concern the integration precision on the trimmed parts, because the Boolean operations (union, subtraction and intersection) are based on the trimming algorithms. Usually, the integration schemes on the trimmed patches are time consuming. Recently, other IGA methods, for example the Additive Schwarz Domain Decomposition Method (ASDDM [9]) and IGAODG[10], are developed, and these methods avoid the integrations on the trimmed patches when the union operation is applied. This paper follows the works of [10] and presents an IGA IIPG method to solve the elasticity problems on the overlapped domains.

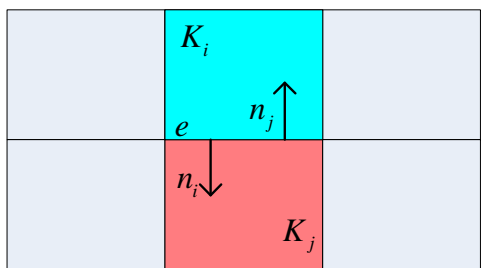

FIGURE I.

A DOMAIN IS MESHED INTO QUADRILATERAL ELEMENTS.
IIPG is one of the DG methods. Simply speaking, for the boundary value problem

$$
\begin{aligned}
& -\Delta u=f \quad \text { in } \Omega \\
& \left.u\right|_{\partial \Omega}=0
\end{aligned}
$$

in which the non-overlapped domain is meshed with quadrilateral elements ( $T_{h}$ in Figure I), the IIPG of problem错误!未找到引用源。 reads as:

Find $u_{h} \in V_{h}$ such that

$$
\begin{aligned}
\int_{T_{h}} f v d x & =\int_{T_{h}} \nabla u_{h} \cdot \nabla v d x-\int_{\Gamma}[v]\left\{\nabla u_{h}\right\} d s \\
& +\int_{\Gamma} \alpha_{e}\left[u_{h}\right][v] d s, \quad \forall v \in V_{h}
\end{aligned}
$$

In (1), for a scalar function $v$ or a vector function $\tau$, the average operator $\{$.$\} and jump operator [.] on the element$ boundaries are defined by

$$
\begin{aligned}
& \text { on } e \not \subset \partial \Omega:\left\{\begin{array}{l}
\{v\}=0.5\left(v_{i}+v_{j}\right),[v]=v_{i} n_{i}+v_{j} n_{j} \\
\{\tau\}=0.5\left(\tau_{i}+\tau_{j}\right),[\tau]=\tau_{i} \cdot n_{i}+\tau_{j} \cdot n_{j}
\end{array}\right. \\
& \text { on } e \subset \partial \Omega:\left\{\begin{array}{l}
{[v]=v n} \\
\{\tau\}=\tau
\end{array}\right.
\end{aligned}
$$

and $\alpha_{e}:=\eta_{e} / h_{e}$ is the penalty coefficient where $\eta_{e}$ is a positive number large enough and $h_{e}$ is the size of the concerned boundary.

For an element $K$, it is indicated that the $\mathrm{C}^{0}-$ and $\mathrm{C}^{1}$ continuities are penalized by $\alpha_{e} v_{K} n_{e}$ and $\frac{v_{K}}{2}$ respectively. For example, the second item $-\int_{\Gamma}[v]\left\{\nabla u_{h}\right\} d s$ indicates the punishment on the $\mathrm{C}^{1}$ - continuity, because

$$
\begin{aligned}
-\int_{e}[v]\left\{\nabla u_{h}\right\} d s & =-\int_{e} v_{i} n_{i} \nabla u_{h, i} d s-\int_{e} v_{j} n_{j} \nabla u_{h, j} d s \\
& +\int_{e} \frac{v_{i}}{2}\left[\nabla u_{h}\right] d s+\int_{e} \frac{v_{j}}{2}\left[\nabla u_{h}\right] d s
\end{aligned} .
$$


In(3), the first two items are naturally brought by the integration by parts formula. If we directly penalize the $\mathrm{C}^{0}$ - and $\mathrm{C}^{1}$ - continuities between the overlapped elements with the same coefficients, we can construct the IIPG method for the overlapped domains.

In this paper, the above mentioned concept is introduced into IGA to solve the elasticity problems defined on the overlapped patches. After the introductions in section I, we briefly introduce the concept of IGA and give some requisite notations for overlapped patches in section II. In section III, we create the discretization scheme for the elasticity equations. In section IV, the convergence property is tested numerically. Finally, the paper is summarized in section V.

\section{IGA AND SOME NOTATIONS}

\section{A. On a Single Patch}

In the following discussions, the hat ' $\wedge$ ' is used to indicate that a function or a space is defined on the parametric domain. Between the parametric patch $\hat{P}$ and the physical patch $P$, a NURBS-based geometry can be seen as a map $F: \hat{P} \rightarrow P$. Therefore, a discrete function space on the physical patch can be defined with

$$
S^{h}(P):=\operatorname{span}\left\{\hat{B}_{i} \circ F^{-1}\right\},
$$

where $\hat{B}_{i}$ is the $i$-th NURBS basis. On a parametric patch, the open knot vectors indicate a mesh, marked as $M_{p r}(\hat{P})$, so that on the physical patch, a physical mesh $M_{p r}(P)$ can be defined by

$$
M_{p r}(P)=\left\{K \mid K=F(\hat{K}), \hat{K} \in M_{p r}(\hat{P})\right\}
$$

Defining a function $\delta_{K}$ by

$$
\delta_{K}=\left\{\begin{array}{ll}
0, & x \notin K \\
1, & \text { else }
\end{array},\right.
$$

the space $S^{h}(P)$ can be further broken into a space $S_{K}^{h}(P)$ where

$$
S_{K}^{h}(P):=\operatorname{span}\left\{v_{K}^{h} \mid v_{K}^{h}=\delta_{K} v^{h}, \forall v^{h} \in S^{h}(P)\right\} .
$$

Functions in $S_{K}^{h}(P)$ may be non-zero out of an element $K$. We use the definition (5) to avoid discussing different continuities of basis between elements. For example, some Bspline basis may be continuous between elements, while the Bezier functions are discontinuous between elements.

\section{B. On United Patches}

We suppose that a parametric patch $\hat{P}$ is precisely meshed into a collection of elements $M_{p r}(\hat{P})=\{\hat{K}\}$. If the CAD model contains multiple patches, we denote by $\hat{T}_{h}=\bigcup_{i} M_{p r}\left(\hat{P}_{i}\right)$ the parametric mesh. Similarly, the physical mesh is defined by $T_{h}=\bigcup_{i} M_{p r}\left(P_{i}\right)$ where

$$
M_{p r}(P)=\left\{K \mid K=F(\hat{K}), \quad \hat{K} \in M_{p r}(\hat{P})\right\}
$$

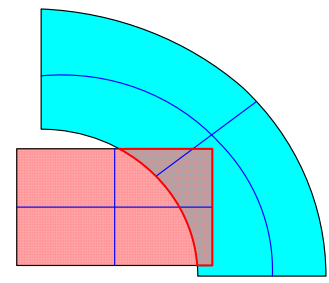

FIGURE II. TWO UNITED PATCHES.

We also suppose that the meshes $T_{h}$ and $\hat{T}_{h}$ are quasiuniform, so that we can use a single symbol $h$ to represents the sizes of both meshes.

If the patches are overlapped, we need three kinds of boundary collections as defined in(6). The first collection $\Gamma_{d}$ contains the external boundaries of the whole domain $\Omega$. The second collection $\Gamma_{e}$ contains all the adjacent boundaries of brother elements in their parent patches. The third collection $\Gamma_{p}$ contains all the external boundaries of patches, which do not belong to $\Gamma_{e} \cup \Gamma_{d}$. In Figure II, boundaries colored red $\subset \Gamma_{p}$, boundaries colored blue $\subset \Gamma_{e}$, and boundaries colored black $\subset \Gamma_{d} \quad$.Finally, we denote by $\Gamma:=\Gamma_{d} \cup \Gamma_{e} \cup \Gamma_{p}=\bigcup_{K}(\partial K)$ the collection of all boundaries of all elements.

$$
\begin{aligned}
& \Gamma_{d}:=\bigcup_{K \in T_{h}}(\partial \Omega \cap \partial K) \\
& \Gamma_{e}:=\bigcup_{P}\left\{e \mid e=\partial K_{i} \cap \partial K_{j}, \forall K_{i}, K_{j} \in M_{p r}(\mathrm{P}), i \neq j\right\} \\
& \Gamma_{p}:=\bigcup_{K}(\partial K) \backslash\left(\Gamma_{e} \cup \Gamma_{d}\right)
\end{aligned}
$$

On the overlapped mesh, we define space $H^{k}\left(T_{h}\right)$ as follows: 1) a function $u$ on each element $K \in T_{h}$ belongs to $\left.H^{k}(K), 2\right)$ the function $u_{K}$ needs not to be continuous with its brother elements, and 3 ) the function $u_{K}$ needs not to be as same as the fields on the other overlapped elements of other parent patches. On $H^{k}\left(T_{h}\right)$, the Sobolev norm is defined by the $L^{2}$ inner product as 


$$
\|u\|_{k, T_{h}}^{2}=\sum_{K \in T_{h}}\left\|u_{K}\right\|_{k, K}^{2}, \quad\left\|u_{K}\right\|_{k, K}^{2}:=\sum_{i=0}^{k}\left\langle D^{i} u, D^{i} u>_{L^{2}(K)} .\right.
$$

Similarly, we define a discrete space $S_{K}^{h}\left(T_{h}\right) \subset H^{k}\left(T_{h}\right)$ by

$$
S_{K}^{h}\left(T_{h}\right)=\operatorname{span}\left\{v_{K}^{h} \mid v_{K}^{h}=\delta_{K} v^{h}, \forall v^{h} \in \forall S^{h}\left(P_{i}\right), \bigcup P_{i}=\Omega\right\}
$$

On the boundary $\partial K_{i}$, for a scalar function $v_{K_{i}} \in S_{K}^{h}\left(T_{h}\right)$, we define its adjacent value $v_{K_{i}, e}$ by

$$
v_{K_{i}, e}(x)=\left\{\begin{array}{l}
v_{K_{j}}(x), \quad \text { on } e \subset \Gamma_{e}, \text { if } x \in \partial K_{i} \cap \partial K_{j} \\
0, \quad \text { on } e \subset \Gamma_{d} \\
v_{K_{j}}(x), \quad \text { on } e \subset \Gamma_{p}, \text { if } x \in \partial K_{i} \cap K_{j}
\end{array} .\right.
$$

If no ambiguity exists, we directly reuse the symbol $S_{K}^{h}\left(T_{h}\right)$ to represent the discrete space of vector (tensor) functions and reuse $v_{K_{i}, e}(x)$ to represent the adjacent value of a vector (tensor) functions.

\section{DisCRETIZATION SCHEME}

In this section, we mainly consider the following linear elasticity problem on domains that are equipped with the ideal, uniform and isotropic materials.

$$
\begin{aligned}
& -\nabla \cdot \sigma=f \\
& u=g_{D} \text { on } \partial \Omega
\end{aligned}
$$

In equation(9), the stress tensor $\sigma(u)$ is defined by

$$
\sigma(u)=\lambda \nabla \cdot u I+2 \mu \varepsilon(u) .
$$

With the Young's modulus $E$ and the Poisson's ratio $v$, the Lame's constants $\lambda$ and $\mu$ in (10) are defined by

$$
\lambda=\frac{E v}{(1+v)(1-2 v)}, \quad \mu=\frac{E}{2(1+v)} .
$$

For simply introduction, we assume the Lame's constants $\lambda$ and $\mu$ are equals to one. In equation(10), the strain tensor $\varepsilon(u)$ is defined by

$$
\varepsilon(u)=\frac{1}{2}\left(\nabla u+\nabla u^{T}\right)
$$

For the non-overlapped case, the IIPG for the problem (9) reads as
Find $u^{h} \in S_{K}^{h}\left(T_{h}\right)$, such that

$$
\begin{aligned}
B_{h}\left(u^{h}, v^{h}\right) & =\int_{T_{h}} f \cdot v^{h} d x, \quad \forall v^{h} \in S_{K}^{h}\left(T_{h}\right) \\
B_{h}\left(u^{h}, v^{h}\right) & :=\sum_{K} \int_{K} \sigma\left(u^{h}\right): \varepsilon\left(v^{h}\right) d x- \\
& \sum_{\partial K} \int_{\partial K}\left\{\sigma\left(u^{h}\right)\right\} n \cdot\left[v^{h}\right] d s+, \\
& \alpha_{e} \sum_{\partial K} \int_{\partial K}\left[v^{h}\right] \cdot\left[u^{h}\right] d s
\end{aligned}
$$

where

$$
\begin{aligned}
& {[u]=u_{K}-u_{K, e} \text {, on } \Gamma \backslash \Gamma_{d}} \\
& {[u]=u, \text { on } \Gamma_{d}}
\end{aligned}
$$

In equation(13), the item $-\int_{\partial K}\left\{\sigma\left(u^{h}\right)\right\} n \cdot\left[v^{h}\right]$ indicates the punishment of the $\mathrm{C}^{1}$ continuity. Firstly, the integration by parts formula for equation(9) reads as

$$
\int_{K} \sigma: \varepsilon-\int_{\partial K} \sigma n \cdot v=\int_{K} f \cdot v d x
$$

Secondly, on an interior boundary $e$ we have

$$
\begin{aligned}
\int_{e}\{\sigma(u)\} n \cdot[v] d s= \\
\int_{e} \sigma\left(u_{h}\right) n_{1} v_{1}+\sigma\left(u_{h}\right) n_{2} v_{2} d s- \\
\int_{e} \frac{1}{2}\left[\sigma\left(u_{h}\right)\right] n_{1} v_{1}+\frac{1}{2}\left[\sigma\left(u_{h}\right)\right] n_{2} v_{2} d s
\end{aligned}
$$

We can see that the last two items penalize the jump $\left[\sigma\left(u_{h}\right)\right]$ on the boundary, and the first two parts are compatible with the formula(14).

Directly penalize the $\mathrm{C}^{0}$ - and $\mathrm{C}^{1}$ - continuities between the overlapped elements with the same coefficients deduced from (13), the IGA IIPG for the elasticity problem reads as:

Find $u^{h} \in S_{K}^{h}\left(T_{h}\right)$, such that 


$$
\begin{aligned}
& B_{T_{h}}^{h}\left(u^{h}, v^{h}\right)=L_{T_{h}}^{h}\left(v^{h}\right), \quad \forall v^{h} \in S_{K}^{h}\left(T_{h}\right) \\
& B_{T_{h}}^{h}\left(u^{h}, v^{h}\right):=\sum_{K} B_{K}^{h}\left(u_{K}^{h}, v_{K}^{h}\right) \\
& B_{K}^{h}\left(u_{K}^{h}, v_{K}^{h}\right):=\int_{K} \sigma\left(u_{K}^{h}\right): \varepsilon\left(v_{K}^{h}\right) d x- \\
& \int_{\partial K} \sigma\left(u_{K}^{h}\right) n \cdot v_{K}^{h} d s+ \\
& \int_{\partial K \cap\left(\Gamma \Gamma_{d}\right)}\left(\sigma\left(u_{K}^{h}\right)-\sigma\left(u_{K, e}^{h}\right)\right) n \cdot \frac{v_{K}^{h}}{2} d s+ \\
& \alpha_{e} \sum_{\partial K} \int_{\partial K} v_{K}^{h} \cdot\left(u_{K}^{h}-u_{K, e}^{h}\right) d s \\
& L_{T_{h}}^{h}\left(v^{h}\right):=\sum_{K} \int_{K} f \cdot v_{K}^{h} d x
\end{aligned}
$$

\section{NuMERICAL EXAMPLES}

In this section, $n$ denotes the variable numbers, and $e_{i}$ denotes the error $\left\|u-u^{h}\right\|_{i, T_{h}}$. On two overlapped models in Figure III, we apply (16) to solve the linear elasticity problem(17) and the convergence results are listed in Figure IV and Figure V.

$$
\begin{gathered}
\nabla \cdot \sigma(u) \\
=\left(\begin{array}{l}
2 \sin (x) \sin (y)-3 y^{2} \cos (x)+2 \cos (x) \\
-4 y \sin (x)-4 \cos (x) \cos (y)
\end{array}\right) \\
\left.u\right|_{\partial \Omega}=\left(\begin{array}{l}
y^{2} \cos (x) \\
\cos (x) \cos (y)
\end{array}\right)
\end{gathered}
$$

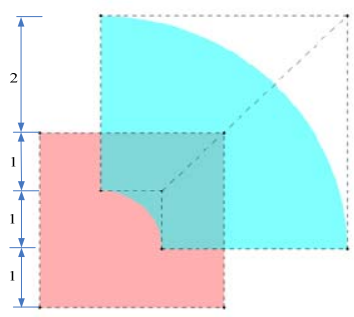

(A)

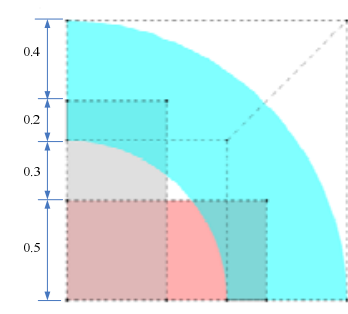

(B)
TWGURE III.
PATCHES, (B) WITH THREE PATCHES

In Figures IV and V, we find that when $p$ changes from 2 to 4 , the IIPG extension gives a convergence rate of $h^{p+1}$ with respect to the norm $\left\|u-u^{h}\right\|_{0, T_{h}}$ and a convergence rate of $h^{p}$ with respect to the norm $\left\|u-u^{h}\right\|_{1, T_{h}}$.

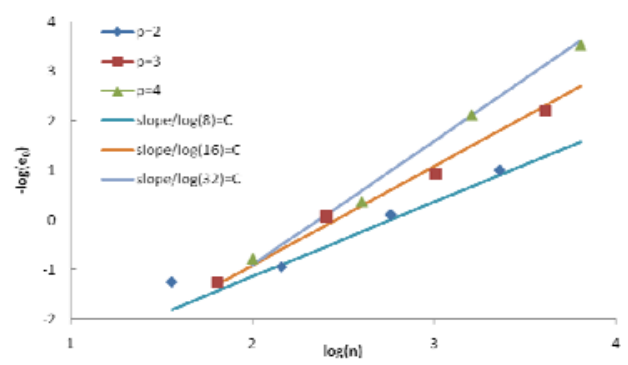

(A) convergence properties on $\left\|u-u^{h}\right\|_{0, T_{h}}$

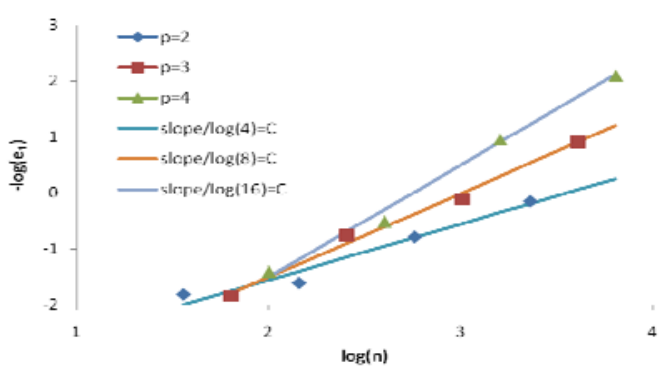

(B) convergence properties on $\left\|u-u^{h}\right\|_{1, T_{h}}$

FIGURE IV. CONVERGENCE PROPERTIES FOR FIGURE III(A)

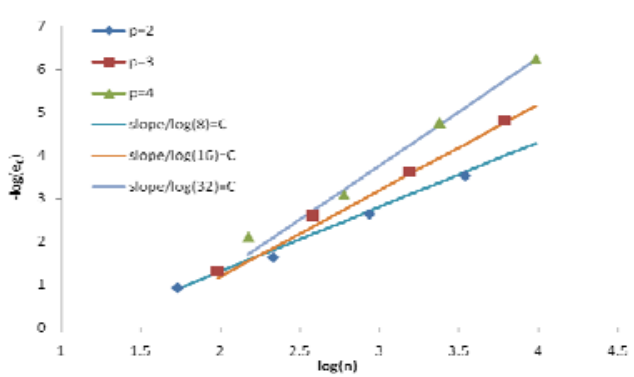

(A) convergence properties on $\left\|u-u^{h}\right\|_{0, T_{h}}$

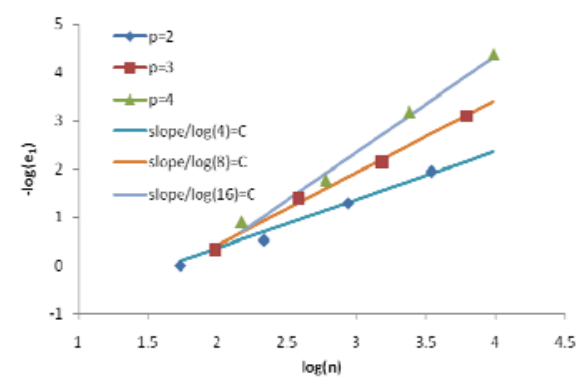

(B) convergence properties on $\left\|u-u^{h}\right\|_{1, T_{h}}$

FIGURE V. CONVERGENCE PROPERTIES FOR FIGURE III(B)

\section{SUMMARY}

By discussing the IIPG of the elasticity problems defined on the non-overlapped domains, and then applying the same punishing coefficients on the overlapped boundaries, the discretization scheme is created to solve the elasticity problems which are defined on the overlapped domains. Numerical results show that the presented IGA IIPG can reach the optimal convergence orders when the patches are overlapped. 


\section{ACKNOWLEDGMENT}

The project is supported by the National Natural Science Fund (Program No.51775445) and the Natural Science Basic Research Plan in Shaanxi Province of China (Program No.2016JM5040).

\section{REFERENCES}

[1] Y. BAZILEVS, L. BEIRÃO DA VEIGA, J. A. COTTRELL, T. J. R. HUGHES, and G. SANGALLI, "ISOGEOMETRIC ANALYSIS: APPROXIMATION, STABILITY AND ERROR ESTIMATES FOR hREFINED MESHES,” Math. Model. Methods Appl. Sci., vol. 16, no. 07, pp. 1031-1090, 2006.

[2] J. A. Cottrell, T. J. R. Hughes, and Y. Bazilevs, Isogeometric Analysis Toward Intergration of CAD and FEA. A John Wiley and Sons, Ltd., 2009.

[3] T. J. R. Hughes, J. A. Cottrell, and Y. Bazilevs, "Isogeometric analysis: CAD, finite elements, NURBS, exact geometry and mesh refinement," Comput. Methods Appl. Mech. Eng., vol. 194, no. 39-41, pp. 4135-4195, 2005.

[4] L. Beirão da Veiga, A. Buffa, J. Rivas, and G. Sangalli, "Some estimates for h-p-k-refinement in Isogeometric Analysis,” Numer. Math., vol. 118, no. 2, pp. 271-305, Oct. 2010.

[5] J. Cottrell, T. J. R. Hughes, and A. Reali, "Studies of refinement and continuity in isogemetric analysis," Comput. Methods Appl. Mech. Eng., vol. 196, pp. 4160-4183, 2007.

[6] D. Natekar, X. Zhang, and G. Subbarayan, "Constructive solid analysis: A hierarchical, geometry-based meshless analysis procedure for integrated design and analysis," CAD Comput. Aided Des., vol. 36, no. 5, pp. 473-486, 2004.

[7] B.-Q. Zuo, Z.-D. Huang, Y.-W. Wang, and Z.-J. Wu, "Isogeometric analysis for CSG models," Comput. Methods Appl. Mech. Engrg., vol. 285, pp. 102-124, 2015.

[8] Y. Wang, Z. Huang, Y. Zheng, and S. Zhang, "Isogeometric analysis for compound B-spline surfaces," Comput. Methods Appl. Mech. Eng., vol. 261-262, pp. 1-15, 2013.

[9] M. Bercovier and I. Soloveichik, "Overlapping non Matching Meshes Domain Decomposition Method in Isogeometric Analysis," arXiv Prepr. arXiv1502.03756, 2015.

[10] H. Zhang, R. Mo, and N. Wan, "An IGA Discontinuous Galerkin Method on the union of overlapped patches," Comput. Methods Appl. Mech. Eng., vol. 326, pp. 446-480, 2017. 